\title{
Definitive three-dimensional high-dose-rate brachytherapy for inoperable endometrial cancer
}

\author{
Lorena Draghini, MD, Ernesto Maranzano, MD, Michelina Casale, PhD, Fabio Trippa, MD, Paola Anselmo, \\ Fabio Arcidiacono, MD, Stefania Fabiani, PhD, Marco Italiani, PhD, Luigia Chirico, MD, Marco Muti, PhD \\ Radiotherapy Oncology Centre, "S. Maria" Hospital, Terni, Italy
}

\begin{abstract}
Purpose: To report our experience on high-dose-rate brachytherapy (HDR-BT) in patients with stage I-III endometrial cancer unfit to surgery.

Material and methods: Seventeen patients underwent HDR-BT as definitive treatment. Median age was 79 years (range, 60-95), median Karnofsky performance status 90\% (range, 60-100). Histology was endometrial adenocarcinoma in $14(82 \%)$, and non-endometrial in $3(18 \%)$ patients. In $15(88 \%)$ patients, clinical stage was I and in remaining $2(12 \%)$ was III. All patients were evaluated with computed tomography (CT) and endometrial biopsy. Using the Fletcher applicator, a CT-based planning HDR-BT was delivered. Local control (LC) was obtained when there was an interruption of vaginal bleeding in absence of CT-imaging progression.

Results: Fourteen patients underwent HDR-BT alone and three external beam radiotherapy (EBRT) combined with HDR-BT. All patients had a clinical LC, after a median follow-up of 53 months (range, 6-131), 3 and 6 years LC rates were $86 \%$ and $69 \%$, respectively. Cancer specific survival (CSS) at 1, 2, and 6 years was $93 \%, 85 \%$, and $85 \%$, respectively. Age, stage, dose, and type of radiotherapy did not result significant prognostic factors for LC and CSS. Only histology significantly influenced LC: for high-risk histology (i.e., non-endometrial carcinoma or grade [G] 3 endometrial adenocarcinoma) LC was 73\% at 1 year and 36\% at 6 years; for low-risk histology (i.e., G1-2 endometrial adenocarcinoma) was $100 \%$ at 1 and 6 years $(p=0.05)$. Two (12\%) patients had G2 acute toxicity and two others $(12 \%)$ G1 late toxicity.

Conclusions: Although some limitations of our analysis (relatively few number of patients recruited, retrospective evaluation, and consequent suboptimal patient selection), it confirms effectiveness and safety of definitive HDR-BT for medically inoperable stage I-III endometrial cancer. The best LC was obtained in stage I low-risk histology.
\end{abstract}

J Contemp Brachytherapy 2017; 9, 2: 118-123

DOl: https://doi.org/10.5114/jcb.2017.67454

Key words: 3D, brachytherapy, endometrial cancer.

\section{Purpose}

Endometrial cancer is the most common gynecological cancer and its incidence is rising; approximately $80 \%$ of cases presents early stage disease with five-year survival rates of over $95 \%$ [1,2]. Standard treatment for localized endometrial cancer is surgery, which includes total abdominal hysterectomy with bilateral salpingo-oophorectomy. Adjuvant radiotherapy and/or chemotherapy are performed based on individual risk factors [2]. However, some patients are medically inoperable due to old age and/or certain comorbidities. Indeed, endometrial cancer frequently concerns older patients with median age of 65 years at diagnosis, and about $10 \%$ of early stage patients have comorbid conditions related to high body mass index, such as cardiovascular disease, obesity-hypoventilation syndrome, and diabetes. These comorbidities are also associated with an increase in iatrogenic toxicity $[3,4]$.
Definitive radiotherapy is an alternative treatment option for this subset of patients not suitable for surgery, and can be performed with brachytherapy (BT) alone or with external beam radiotherapy (EBRT) plus BT. Literature data, summarized in Schwarz et al. consensus statement on brachytherapy for medically inoperable endometrial cancer, reports 5-year disease-free survival (DFS), ranging from $72 \%$ to $95 \%$, and local control (LC) between $71 \%$ and $93 \%$ in patients treated with definitive radiotherapy. In the majority of these studies, EBRT and BT were combined without three-dimensional (3D) image-based planning system. Moreover, old and obese patients were often not compliant to EBRT for high number of fractions and toxicity risk [5].

With 3D high-dose-rate BT (HDR-BT) we can apply a higher dose to the target volume, improving LC limiting dose at organs at risk (OAR). In this way, HDR-BT alone can be an adequate therapeutic tool for old and obese
Address for correspondence: Ernesto Maranzano, MD, Director of Department of Oncology and Chair of Radiotherapy Oncology Centre, "S. Maria" Hospital, Via T. di Joannuccio, 105100 Terni, Italy, phone: +390744 205729, fax: +39 0744 205034, « e-mail: e.maranzano@aospterni.it
Received: 17.11.2016

Accepted: 21.03 .2017

Published: 28.04.2017 
patients with localized endometrial cancer. Our experience on 3D HDR-BT with or without EBRT in patients with stage I-III endometrial cancer unfit to surgery is reported in this paper.

\section{Material and methods}

Between March 2005 and April 2016, 443 patients with endometrial cancer were treated at our institution: 426 (96\%) underwent surgery and adjuvant radiotherapy, 14 (3\%) HDR-BT alone, and $3(1 \%)$ EBRT plus HDR-BT without surgery. We evaluated these last 17 patients inoperable for comorbidities and/or old age, and submitted to radiotherapy without surgery. At diagnosis, patients were assessed using clinical examination, computed tomography $(\mathrm{CT})$, and endometrial biopsy. Although pelvic magnetic resonance imaging (MRI) should have been ideally done in all cases to evaluate disease extension and treatment response, only two patients were assessed with MRI. Reasons conditioning this choice were as follows: two patients were stage III at CT for vaginal or pelvic lymph nodes extension, eight very old patients (81-95 years) refused MRI, five had contraindications (e.g., metal leg prosthesis). Non-endometrial carcinoma or grade G3 endometrial adenocarcinoma were considered as highrisk histology, in the other cases, as low-risk histology.

The EBRT was delivered with 3D technique with a CT-based planning system. Oral contrast was given to visualize small intestine, and CT was done in prone position with empty rectum and full bladder. The clinical target volume (CTV) included the entire uterus, upper half of the vagina, lower common iliac, external and
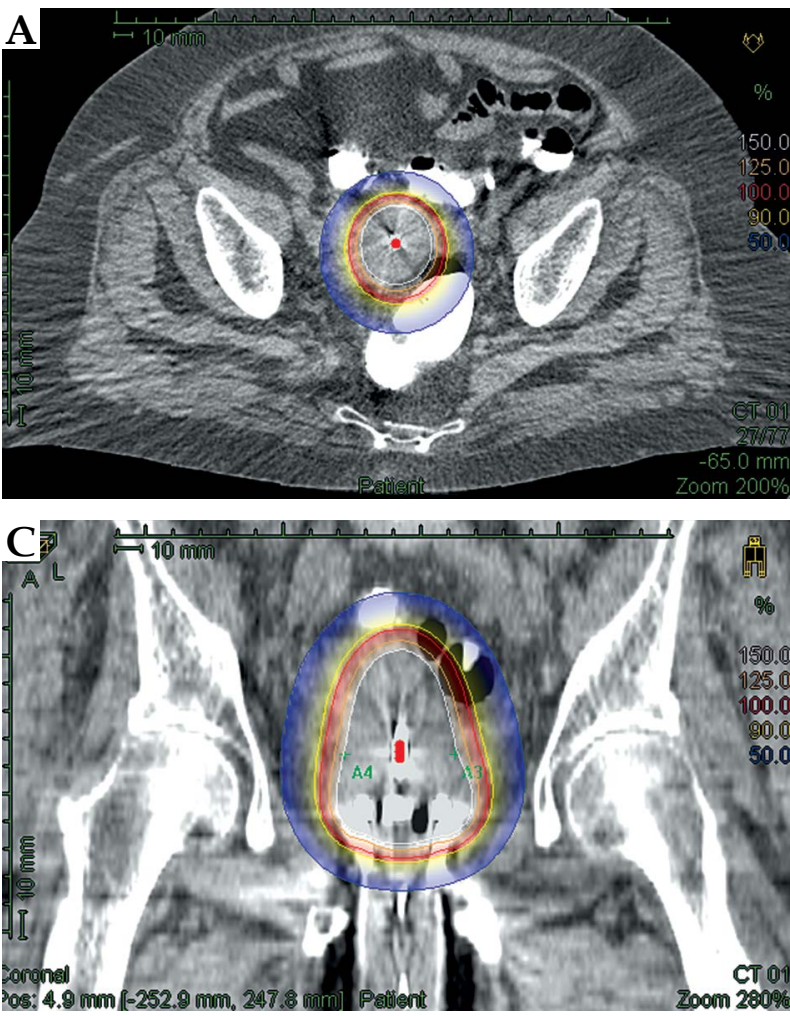

Fig. 1. Representative dose distribution of brachytherapy internal iliac, obturator and presacral nodes. For EBRT, CTV was defined by a $7 \mathrm{~mm}$ expansion of nodal regions with subtraction of the pelvic bones, femoral heads, and vertebral bodies; then a margin of generally $5 \mathrm{~mm}$ in all directions was added to CTV to obtain PTV (planning target volume).

For HDR-BT, a CT-based planning was performed using a single uterine applicator, and then orthogonal X-ray films were completed each day to verify the appropriateness of placement. In our series, we used the Fletcher applicator, and patients were selected on the basis of an adequate coverage of the CTV with a single uterine applicator. Figure 1 shows a representative dosimetric picture of dose distribution.

The brachytherapy CTV included uterus, cervix, and upper vagina; prescription and optimization were performed on CTV based on uterine size, configuration, and disease location. Treatment plans were generated on the Plato Brachytherapy Planning System, version 14.2.6 (Nucletron, an Elekta company, Elekta AB, Stockholm, Sweden) or Oncentra Brachytherapy Planning System, version 4.1.2 (Nucletron). High-dose-rate brachytherapy was administered 3 times a week; fractionation schemes were selected taking into account equivalent dose, OAR constrains, patient compliance, and previous EBRT dose.

Brachytherapy dose was prescribed to the volume contoured at CT and defined by several points placed at the CTV surface. Initially, standard weighting was placed at each source position, and then manually adjusted to adequately cover the CTV and reduce OAR doses. The volumes of organs at risk, including rectum, femoral heads, small bowel, and bladder were also contoured. Plan opti-
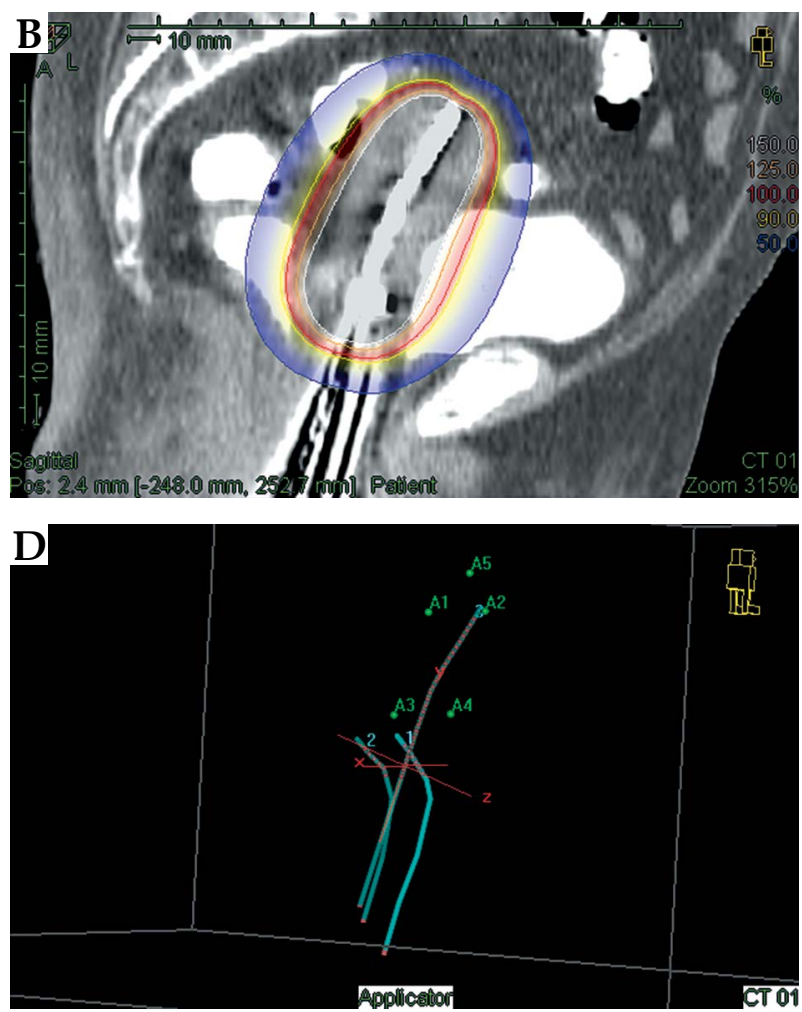
Table 1. Administered doses

\begin{tabular}{lll} 
& Number of patients (\%) & $\mathrm{EQD}_{2}\left(\alpha / \beta_{10}\right)$ \\
\hline HDR-BT & $1(6)$ & $20 \mathrm{~Gy}$ \\
\hline $2 \times 7 \mathrm{~Gy}^{*}$ & $1(6)$ & $19 \mathrm{~Gy}$ \\
\hline $3 \times 5 \mathrm{~Gy}^{*}$ & $1(6)$ & $24 \mathrm{~Gy}$ \\
\hline $3 \times 6 \mathrm{~Gy}^{*}$ & $1(6)$ & $24 \mathrm{~Gy}$ \\
\hline $3 \times 6 \mathrm{~Gy}$ & $2(12)$ & $42 \mathrm{~Gy}$ \\
\hline $3 \times 7 \mathrm{~Gy}$ & $3(18)$ & $36 \mathrm{~Gy}$ \\
\hline $3 \times 8 \mathrm{~Gy}$ & $2(12)$ & $40 \mathrm{~Gy}$ \\
\hline $4 \times 7 \mathrm{~Gy}$ & $5(28)$ & $40 \mathrm{~Gy}$ \\
\hline $5 \times 6 \mathrm{~Gy}$ & $1(6)$ & $44 \mathrm{~Gy}$ \\
\hline $7 \times 5 \mathrm{~Gy}$ & $1(6)$ & \\
\hline EBRT & $2(12)$ & $56 \mathrm{~Gy}$ \\
\hline $23 \times 2 \mathrm{~Gy}^{*}$ & $50 \mathrm{~Gy}$
\end{tabular}

$H D R-B T$ - high-dose-rate brachytherapy, $E Q D_{2}$ - equivalent dose of 2 Gy per fraction, EBRT - external beam radiotherapy

*Three patients submitted to external beam radiotherapy combined with brachytherapy

mization parameters included: $95 \%$ of the PTV to receive 95\% of the prescription dose for EBRT. Brachytherapy CTV received at least $90 \%$ of the prescription dose, $<50 \%$ of the rectum to receive $50 \mathrm{~Gy},<35 \%$ of the rectum to receive $60 \mathrm{~Gy}$, D-max to femoral heads and small bowel $<55 \mathrm{~Gy}$, and $<50 \%$ of the bladder to receive $65 \mathrm{~Gy}$. For tolerance, dose concerning late effects $\alpha / \beta$ ratio of 3 was used in the calculation of the equivalent dose in 2 Gy fraction $\left(\mathrm{EQD}_{2}\right)$ to OAR. For combined treatment cumulative (i.e., EBRT plus BT), the dose was calculated [6].

Follow-up was performed with physical examination and CT while MRI was performed only in two cases. Considering that our patients were generally unfit for a MRI evaluation, LC was defined as an interruption of vaginal bleeding in absence of CT-imaging progression. In case

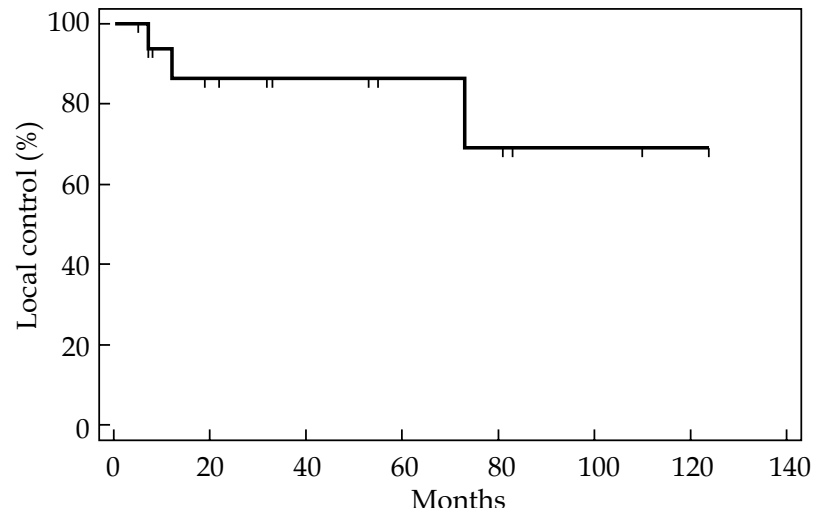

Number of patients

$\begin{array}{llllllll}17 & 10 & 7 & 5 & 4 & 2 & 1 & 0\end{array}$

Fig. 2. Local control probability for all patients of recurrent bleeding and/or CT-imaging progression, a confirmatory endometrial biopsy was done to diagnose a relapse. Common Terminology Criteria for Adverse

Events (CTAEC) version 4.03 was used to grade toxicities.

We performed a retrospective analysis. Study endpoints were LC, overall survival (OS; time from radiotherapy to the last follow-up examination or death event), cancer specific survival (CSS; survival specifically related to the primary tumor), and DFS (survival until date of disease progression). The prognostic impact of parameters such as age, histology, stage, dose, and type of radiotherapy were also assessed. Statistical analysis was performed using a software package (MedCalc 11.1 Broekstraat 52, B-9030 Mariakerke, Belgium) and the Kaplan-Meier product-limit method [7]. The evidence of statistically significant differences in LC, OS, and CSS between subgroups was assessed with the log-rank test. A value of $p<0.05$ (two tailed) was considered statistically significant.

\section{Results}

\section{Patient and treatment characteristics}

Seventeen patients with endometrial cancer were treated at our institution with radiotherapy without surgery. Median age was 79 years (range, 60-95), and median Karnofsky performance status (KPS) 90\% (range, 60-100). Histology was G1-2 endometrial adenocarcinoma in 9 (53\%), G3 endometrial adenocarcinoma in 5 (29\%), and non-endometrial carcinoma in $3(18 \%)$ patients (sero-papillar carcinoma in 1 and clear cell carcinoma in 2 patients). Therefore, $9(53 \%)$ patients were considered with lowrisk histology and $8(47 \%)$ with high-risk histology. In $15(88 \%)$ patients, clinical stage was I, and in remaining $2(12 \%)$ were stage IIIB for vaginal and IIIC1 for pelvic lymph nodes extension, respectively.

Fourteen patients underwent HDR-BT alone, 13 had endometrial adenocarcinoma (nine G1-2, and four G3), and one, a clear cell carcinoma. The EBRT and HDR-BT were performed in 3 patients, one with pelvic lymph nodes extension, another with vaginal extension and sero-papillar histology, and last one with clear cell histology. These 3 patients received EBRT not only because of high-risk disease, but also on the basis of their compliance. Remaining high-risk patients were judged as unfit for EBRT, due to lower KPS, old age, and/or obesity.

\section{Efficacy results}

Median brachytherapy CTV was 80.6 cc (range, 50270), administered doses are shown in Table 1 . After a median follow-up of 53 months (range, 6-131), all (100\%) patients had a clinical LC with resolution of vaginal bleeding. At 3 and 6 years, LC rates were $86 \%$ and $69 \%$, respectively, whereas median LC was not reached (Figure 2). In particular, 3 patients (18\%) had local relapse documented by imaging and endometrial biopsy (in one case uterine relapse; in another case uterine and nodal relapse; in the last case, uterine, nodal, and peritoneal relapse). All these 3 patients died from progression of the disease.

Median OS was 80 months (range, 6-131). Thirteen patients $(77 \%)$ are alive, ten without evident disease, remain- 


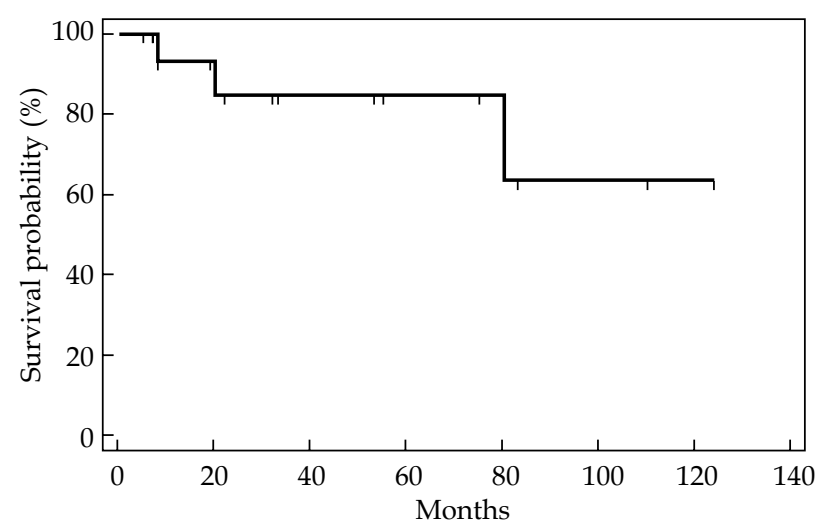

Number of patients

$\begin{array}{llllllll}17 & 10 & 7 & 5 & 3 & 2 & 1 & 0\end{array}$

Fig. 3. Cancer specific survival probability for all patients

ing 3 with a second cancer. Four $(23 \%)$ patients died, two for local and systemic progression, one for local relapse, one for senile marasmus. Cancer specific survival at 1, 2, and 6 years was 93\%,85\%,85\%, respectively (Figure 3). Moreover, DFS at 1, 2, and 6 years was $86 \%, 79 \%$, and $63 \%$, respectively, with a median not reached (Figure 4 ). At univariate analysis, age, stage, dose, and type of radiotherapy did not result significant prognostic factors for LC, CSS, and DFS. For 1-year LC, there was a trend in favor of stage I ( $91 \%$ for stage I, and $50 \%$ for stage III), but this difference did not achieve statistical significance $(p=0.06)$. However, histology significantly influenced LC. Patients with low-risk histology compared to those with high-risk histology had a better LC at first and $6^{\text {th }}$ year $(100 \%$ and $100 \%$, versus $73 \%$ and $36 \%$, respectively; $p=0.05)$. Nevertheless, median duration of LC was also quite good for high-risk histology patients (i.e., 73 months), while for low-risk histology, the median value was not reached (Figure 5).

\section{Toxicity}

Only patients submitted to EBRT in combination with HDR-BT developed iatrogenic toxicity. Acute toxicity was registered in $2(12 \%)$ patients: G2 nausea and G2 proctitis in one case $(6 \%)$, G2 diarrhea, G2 anemia, and G2 proctitis in another $(6 \%)$ case. Two patients $(12 \%)$ had G1 late rectal bleeding.

\section{Discussion}

Definitive radiotherapy is a treatment option for medically inoperable endometrial cancer, and can be performed with HDR-BT alone or EBRT combined with HDR-BT [5]. In the past, HDR-BT for endometrial cancer was done without 3D-image-based planning system with a lack of accurate evaluation of CTV and OAR $[5,8]$. In this setting, differently from cervical cancer, the benefit of 3D HDR-BT treatment plan was probably underestimated, and only few studies on 3D HDR-BT for endometrial cancer has been published $[4,5,9,10,11,12,13,14,15]$. Table 2 summarizes studies of the last 10 years reporting definitive 3D HDR-BT with or

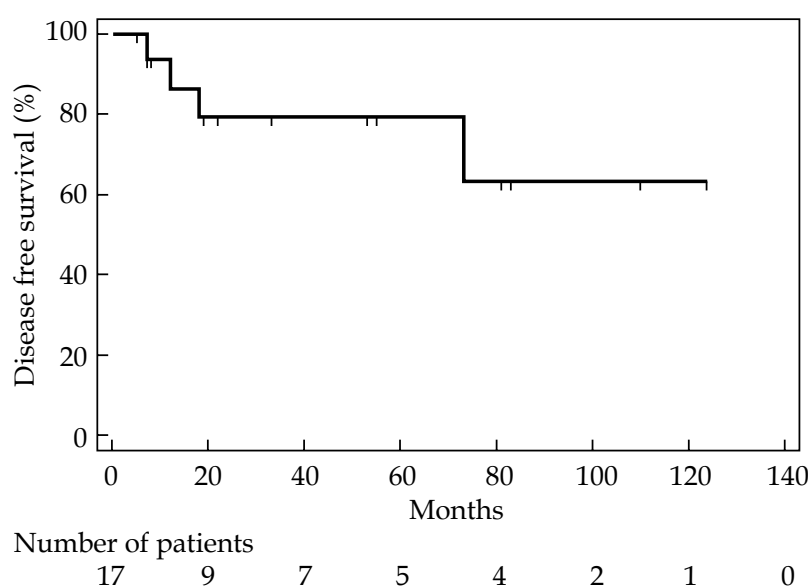

Fig. 4. Disease free survival probability for all patients

without EBRT in medically inoperable endometrial cancer. Compared with other published data, we had a lower number of patients, but a longer median follow-up (53 months) and a higher percentage of patients submitted to HDR-BT alone.

Table 2 shows also that duration of local control was generally high, varying from $80 \%$ to $100 \%$. In our experience, LC resulted marginally lesser (69\% at 6 years), possibly for the presence of high-risk factors (such as highrisk histology and/or locally advanced stage III) in the 3 relapsed patients. Prescribed doses to target volume were generally higher when EBRT was administered in combination with HDR-BT, both in our experience and in other reported studies $[4,10,12,13,14,15]$. In medically inoperable patients, American Brachytherapy Society recommends more aggressive treatment for more aggressive disease (such as high-risk histology and/or locally advanced stage III) with a combination of EBRT and HDR-BT, in order to apply higher doses in larger volumes. However, these recommendations are not always applicable [5]. The choice in favor of HDR-BT alone in clinical practice is often related to a patient selection on the basis of general condition, age,

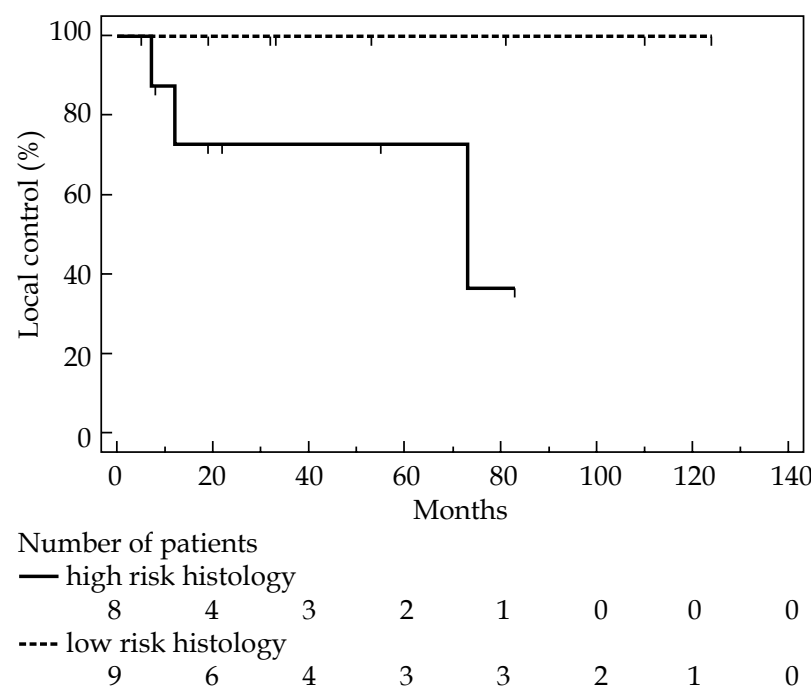

Fig. 5. Local control probability according to histology 
Table 2. Available literature on definitive radiotherapy for endometrial cancer using three- dimensional high-doserate brachytherapy \pm external beam radiotherapy

\begin{tabular}{|c|c|c|c|c|c|c|c|}
\hline $\begin{array}{l}\text { Author } \\
\text { (reference) }\end{array}$ & $\begin{array}{l}\text { Patient } \\
\text { No. }\end{array}$ & Stage & $\begin{array}{c}\text { Type of radiotherapy } \\
\text { and doses }\end{array}$ & $\begin{array}{l}\text { Median } \\
\text { follow-up } \\
\text { (months) }\end{array}$ & $\begin{array}{l}\text { Local control } \\
\text { rates }\end{array}$ & $\begin{array}{l}\text { Cancer specific } \\
\text { survival }\end{array}$ & $\begin{array}{c}\text { Grade } 3-4 \\
\text { toxicity } \\
(\%) \\
\end{array}$ \\
\hline $\begin{array}{l}\text { Weitmann } \\
\text { et al. } 2005 \text { [13] }\end{array}$ & 13 & $|-| \mid$ & HDR-BT 5-7 x 6-7 Gy & 47 & $100 \%$ at 4 years & $100 \%$ at 5 years & 0 \\
\hline \multirow{2}{*}{$\begin{array}{l}\text { Coon et al. } \\
2008[12]\end{array}$} & 35 & \multirow[t]{2}{*}{$|-|||$} & EBRT 45 Gy + HDR-BT 5 x 4 Gy & \multirow[t]{2}{*}{33} & \multirow[t]{2}{*}{$93 \%$ at 3 years } & \multirow{2}{*}{$\begin{array}{l}93 \% \text { at } 3 \text { years } \\
87 \% \text { at } 5 \text { years }\end{array}$} & \multirow[t]{2}{*}{13} \\
\hline & 14 & & HDR-BT $5 \times 7$ Gy & & & & \\
\hline \multirow{2}{*}{$\begin{array}{l}\text { Ohkubo } \\
\text { et al. } 2011[15]\end{array}$} & 9 & \multirow[t]{2}{*}{$|-| \mid$} & EBRT 30.6 Gy + HDR-BT 4 x 6 Gy & \multirow[t]{2}{*}{52} & \multirow[t]{2}{*}{$100 \%$ at 5 years } & \multirow[t]{2}{*}{$100 \%$ at 5 years } & \multirow[t]{2}{*}{0} \\
\hline & 1 & & HDR-BT $4 \times 6$ Gy & & & & \\
\hline \multirow{2}{*}{$\begin{array}{l}\text { Gill et al. } 2014 \\
\text { [10] }\end{array}$} & 18 & \multirow[t]{2}{*}{1} & EBRT45 Gy + HDR-BT 4-5 x 5 Gy & \multirow[t]{2}{*}{15} & \multirow[t]{2}{*}{$91 \%$ at 2 years } & \multirow[t]{2}{*}{$100 \%$} & \multirow[t]{2}{*}{0} \\
\hline & 20 & & HDR-BT 5-6 x 7 Gy & & & & \\
\hline \multirow{3}{*}{$\begin{array}{l}\text { Acharya } \\
\text { et al. } 2015 \text { [14] }\end{array}$} & 260 & \multirow[t]{3}{*}{1} & EBRT & \multirow[t]{3}{*}{ n.a. } & \multirow[t]{3}{*}{ n.a. } & $74 \%$ at 3 years & \multirow[t]{3}{*}{ n.a. } \\
\hline & 144 & & $E B R T+H D R-B T$ & & & $82 \%$ at 3 years & \\
\hline & 46 & & HDR-BT & & & & \\
\hline \multirow{2}{*}{$\begin{array}{l}\text { Acharya et al. } \\
2016[4]\end{array}$} & 15 & \multirow[t]{2}{*}{$|-|||$} & EBRT48-50.4 Gy + HDR-BT 6 x 3.75 Gy & \multirow[t]{2}{*}{29} & \multirow[t]{2}{*}{$80 \%$ at 12 years } & \multirow[t]{2}{*}{$65 \% *$ at 2 years } & \multirow[t]{2}{*}{4.6} \\
\hline & 28 & & HDR-BT 6 × 6 Gy & & & & \\
\hline \multirow[t]{2}{*}{ This report } & 3 & \multirow[t]{2}{*}{$|-| I \mid$} & EBRT 46-50 Gy + HDR-BT 3-7 x 5-8 Gy & \multirow[t]{2}{*}{53} & \multirow[t]{2}{*}{$69 \%$ at 6 years } & \multirow[t]{2}{*}{$85 \%$ at 6 years } & \multirow[t]{2}{*}{0} \\
\hline & 14 & & HDR-BT 3-7 x 5-8 Gy & & & & \\
\hline
\end{tabular}

$H D R-B T$ - high-dose-rate brachytherapy, EBRT - external beam radiotherapy, n.a. - not available

${ }^{*}$ Overall survival

and body mass. In a population of patients already considered inoperable, there is always someone who cannot receive even EBRT due to bad performance status, old age, and/ or obesity. In our series, the old age of patients (median, 79 years) conditioned the choice of HDR-BT alone in the majority of cases (14 of $17 ; 82 \%)$.

While the treatment was generally not aggressive, long-term outcome of our patients was effective and safe with a median OS of 80 months, 6-year CSS of $85 \%$, and no grade 3-4 iatrogenic toxicity. Apart from some recent experience, in which 5-year CSS reached 100\% [13,15], our results were similar to those reported by authors, which recruited not only stage I patients with inoperable endometrial cancer, but also stage II-III disease [4,5,12].

Stage, age, histology, dose, and type of radiotherapy were described in literature as factors, which can condition prognosis in this setting of patients $[2,4,11,14,16,17,18]$. In our analysis, only histology resulted as a statistical significant prognostic factor for LC, that was at 1 and 6 years: $73 \%$ and $36 \%$ for high-risk histology, $100 \%$ and $100 \%$ for low-risk histology, respectively $(p=0.05)$. Also the stage seemed to influence LC at 1 year: $50 \%$ for stage III, and $91 \%$ for stage I, but this difference did not reach statistical significance $(p=0.06)$. Relatively low number of patients could be the reason for lack of difference.

It is worth to notice that acute and late G1-2 toxicities were registered in a minority of cases $(12 \%)$, and were related to EBRT combined with HDR-BT [19]. In general, the treatment was well tolerated despite old patient age.
Although some limitations of our analysis (relatively few number of patients recruited, retrospective evaluation, and consequent suboptimal patient selection), it confirms effectiveness and safety of definitive HDR-BT for medically inoperable stage I-III endometrial cancer. The best LC was obtained in stage I low-risk histology.

\section{Disclosure}

Authors report no conflict of interest.

\section{References}

1. Fader AN, Arriba LN, Frasure HE, von Gruenigen VE. Endometrial cancer and obesity: epidemiology, biomarkers, prevention and survivorship. Gynecol Oncol 2009; 114: 121-127.

2. Colombo N, Cruetzberg C, Amant F et al. ESMO-ESGOESTRO consensus conference on endometrial cancer diagnosis treatment and follow-up. Int J Gynecol Cancer 2016; 26: 2-30.

3. Wegener RE, Beriwal S, Heron DE et al. Definitive radiation therapy for endometrial cancer in medically inoperable elderly patients. Brachytherapy 2010; 9: 260-265.

4. Acharya S, Esthappan J, Badiyan S et al. Medically inoperable endometrial cancer in patients with a high body mass index (BMI): Patterns of failure after 3D image-based high dose rate (HDR) brachytherapy. Radiother Oncol 2016; 118: 167-172.

5. Schwarz JK, Beriwal S, Esthappan J et al. Consensus statement for brachytherapy for the treatment of medically inoperable endometrial cancer. Brachytherapy 2015; 14: 587-599.

6. Liu L, Bassano DA, Prasad SC et al. The linear-quadratic model and fractionated stereotactic radiotherapy. Int J Radiat Oncol Biol Phys 2003; 57: 827-832.

7. Kaplan EL, Meier P. Nonparametric estimation from incomplete observations. J Am Stat Assoc 1958; 53: 457-481. 
8. Dankulchai P, Petsuksiri J, Chansilpa Y, Hoskin PJ. Imageguided high-dose-rate brachytherapy in inoperable endometrial cancer. Br J Radiol 2014; 87: 20140018.

9. Rijkmans EC, Nout RA, Rutten IH et al. Improved survival of patients with cervical cancer treated with image-guided brachytherapy compared with conventional brachytherapy. Gynecol Oncol 2014; 135: 231-238.

10. Gill BS, Kim H, Houser C et al. Image-based three-dimensional conformal brachytherapy for medically inoperable endometrial carcinoma. Brachytherapy 2014; 13: 542-547.

11. Gill BS, Chapman BV, Hansen KJ et al. Primary radiotherapy for nonsurgically managed Stage I endometrial cancer: Utilization and impact of brachytherapy. Brachytherapy 2015; 14: 373-379.

12. Coon D, Beriwal S, Heron DE et al. High-dose-rate Rotte "Y" applicator brachytherapy for definitive treatment of medically inoperable endometrial cancer: 10-year results. Int J Radiat Oncol Biol Phys 2008; 71: 779-783.

13. Weitmann HD, Pötter R, Waldhäusl C et al. Pilot study in the treatment of endometrial carcinoma with 3D image-based high-dose-rate brachytherapy using modified Heyman packing: clinical experience and dose-volume histogram analysis. Int J Radiat Oncol Biol Phys 2005; 62: 468-478.

14. Acharya S, Perkins SM, DeWees T et al. Brachytherapy is associated with improved survival in inoperable stage I endometrial adenocarcinoma: a population-based analysis. Int J Radiat Oncol Biol Phys 2015; 93: 649-657.

15. Ohkubo Y, Kato S, Kiyohara $\mathrm{H}$ et al. Dose volume analysis of radiotherapy for inoperable patients with stage I-II endometrial carcinoma. J Radiat Res 2011; 52: 666-673.

16. Niazi TM, Souhami L, Portelance L et al. Long-term results of high-dose-rate brachytherapy in the primary treatment of medically inoperable stage I-II endometrial carcinoma. Int J Radiat Oncol Biol Phys 2005; 63: 1108-1113.

17. Huddleston A, Zhen S, Qi L et al. The impact of vaginal brachytherapy boost to pelvic radiation in stage III endometrial cancer. J Contemp Brachytherapy 2015; 7: 122-127.

18. Thompson SR, Delaney GP, Gabriel GS, Barton MB. Patterns of care study of brachytherapy in New South Wales: malignancies of the uterine corpus. J Contemp Brachytherapy 2015; 7: $224-230$

19. Guler OC, Onal C, Acibuci I. Effects of bladder distension on dose distribution of vaginal vault brachytherapy in patients with endometrial cancer. J Contemp Brachytherapy 2015; 6: $371-376$ 